\title{
THE THEORY OF THE EARTH'S ORIENTATION, WITH SOME NEW RESULTS FOR NUTATION
}

\author{
John M. Wahr \\ Department of Physics and \\ Cooperative Institute for Research in Environmental Sciences \\ Campus Box 390 \\ University of Colorado \\ Boulder, Colorado 80309
}

\begin{abstract}
The rotation of the earth is variable at a number of time scales, from a few days to thousands of years and longer. Variations occur in the rotation rate, in the geographical position of the rotation axis (referred to as polar motion), and in the position of the axis relative to inertial space (referred to as nutation and precession). The interpretations of the various observations have implications for the dynamical behavior and structure of the earth's deep interior, and for various aspects of meteorology and oceanography. These are reviewed below. Also included is an Appendix describing a model of the diurnal resonance in nutation for a non-hydrostatically pre-stressed earth.
\end{abstract}

\section{INTRODUCTION}

The earth's rotation is not constant. Instead, there are variations both in the rate of rotation and in the position of the rotation axis. Variations in the rate of rotation affect the length of a day (lod), where "a day" is defined in terms of the overhead transits of celestial objects. The time integral of the lod variability is proportional to perturbations in Universal Time.

To describe motion of the rotation axis, it is useful to differentiate between (1) geographical motion: motion with respect to fixed points on the earth's surface; and (2) inertial space motion: motion with respect to celestial objects. Geographical motion of the axis is referred to as polar motion or, sometimes, wobble. Inertial space motion is called nutation or, in a special case, precession. In a sense, this differentiation is artificial because there cannot be inertial space motion without geographical motion, and vice versa. Still, the distinction is useful because most disturbances of the pole can be characterized as either predominately nutation or predominately polar motion, depending on the frequency of the disturbance.

For example, any perturbation of the axis with a period much longer than one day as seen from space, will produce motion which is mostly nutation with very little polar motion: the rotation axis and fixed terrestrial points move almost together at long periods. This means that the external gravitational torques on the earth from the sun and moon will cause motion which is predominately nutation, since those torques vary with the various orbital periods characterizing the lunar and solar orbits, and those 
periods are all much longer than one day. Note that to an observer fixed to the rotating earth, the periods of those torques, and so of the corresponding axis motion, will be close to one day. On the other hand, disturbances which perturb the pole at periods much longer than one day as seen from the rotating earth, cause mostly polar motion with very little nutation: the rotation axis doesn't move much in inertial space, but fixed terrestrial points do. This means that processes which originate within the earth and which have time scales much longer than a day, cause mostly polar motion.

I will discuss, below, the observed variability in the lod, in polar motion, and in nutation, together with the potential geophysical implications. I will concentrate on the shorter period variability (periods of a few years and less), with the rationale that because of the relatively short data span of high quality VLBI observations, those observations through the near future will mostly impact our understanding of the short period variability. More detailed discussions can be found in Munk and MacDonald (1975), Lambeck (1980), and Rochester (1984).

\section{LENGTH OF DAY}

Let the unperturbed rotation vector of the earth be in the $\hat{\mathbf{z}}$ direction, with amplitude $\Omega$ equal to one cycle per sidereal day. Suppose the rotation rate of the mantle is perturbed, so that its time-dependent amplitude is $\Omega\left(1+\mathrm{m}_{3}(\mathrm{t})\right)$. Suppose we apply an external torque, $L_{3}(t)$, to the mantle along the $\hat{z}$ axis. Let $C$ be the unperturbed value of the earth's principal moment of inertia along the $\hat{\mathbf{z}}$ axis. Suppose we perturb the moment of inertia, so that its value at time $\mathrm{t}$ is $\mathrm{C}+\Delta \mathrm{c}(\mathrm{t})$. Then the angular momentum equation for the mantle reduces to

$$
\frac{\mathrm{d}}{\mathrm{dt}} \mathrm{m}_{3}=\frac{\mathrm{L}_{3}}{\mathrm{C} \Omega}-\frac{1}{\mathrm{C}} \frac{\mathrm{d}}{\mathrm{dt}} \Delta \mathrm{c}
$$

As equation (1) demonstrates, the earth's rotation rate can be perturbed either by applying an external torque to the earth, or by changing the earth's inertia tensor.

\subsection{Long Period Variability}

Historic eclipse records indicate that the lod has been increasing by about 2 msec per century over at least the last 2500 years. This linear increase is mostly due to lunar and solar torques $\left(\mathrm{L}_{3}\right.$ in $\left.(1)\right)$ on the earth which tend to slow the earth's rotation and which arise because of dissipation of tidal energy in the oceans. However, these tidal torques are about $25 \%$ larger than those implied by the eclipse data. The discrepancy is due to the effects of post-glacial rebound. Mass continues to flow laterally into the region of the mantle beneath Hudson's Bay as the earth adjusts to the melting of the Canadian ice load at the end of the last ice age. As a result, the earth's polar moment of inertia, $\Delta c$, is decreasing and this tends to decrease the lod, partially offsetting the effects of tidal dissipation.

Telescope data over the last few hundred years demonstrate that there are also irregular fluctuations in the lod with periods of a few decades and amplitudes of up to 45 msec. These are presumably caused by the exchange of angular momentum between the earth's mantle and fluid core. The torques responsible for the exchange of angular momentum could be due either to electromagnetic or to topographic coupling. As the VLBI data span becomes longer so that VLBI is able to resolve the long period 
variability, the results may help increase our understanding of the dynamical processes and the material and structural properties of the core and lower mantle.

\subsection{Short-Period Variability}

There are two important excitation sources which cause variations in the lod at periods of a few years and less: tides, particularly in the solid earth; and the exchange of angular momentum between the earth's mantle and the atmosphere.

Tidal deformation in the earth causes the principal polar moment of inertia, and so the lod, to vary at the periods of the long period tides. Particularly important perturbations occur at periods of 1 year, 6 months, 1 month, and 2 weeks. The amplitudes of these lod variations depend on how easily the earth deforms at these periods. The deformation can be calculated by solving a set of partial differential equations which represent the dynamical response of the earth to tidal forcing. Any such model requires, as input, a set of numerical parameters describing the earth's material properties. The parameters which describe the elastic behavior of the earth are well known from seismology. But, the parameters which describe the departure of the earth from perfect elasticity are not well constrained. Seismic observations are sensitive to anelastic effects, but those effects are apt to be substantially different at the long tidal periods than at the much shorter periods (seconds to minutes) characteristic of seismic phenomena. In fact, one of the important problems in geophysics is to understand more fully the physical processes responsible for the earth's anelasticity. Tidal lod observations can help in this regard, since they offer information on anelasticity in a unique frequency regime.

The 1 month and 2 week tidal variations in the lod have amplitudes of about .2 msec and $.4 \mathrm{msec}$, respectively. The effects of anelasticity on the amplitudes are probably $1 \%-5 \%$ of these numbers (Wahr and Bergen, 1986), so they perturb the lod at the $.01 \mathrm{msec}$ level. Merriam (1985) used BIH optical results for the tidal variations to constrain the anelasticity. Results based on VLBI observations should give improved estimates within the near future, although the interpretation of any data is limited by the accuracy with which the effects on the lod of ocean tides and atmospheric fluctuations at these periods can be removed from the data. In fact, this is why the observed 6 month and 1 year amplitudes can not be used to constrain mantle anelasticity: the seasonal effects of the atmosphere are large, comparable to the tidal effect at 6 months and about 10 times larger at 1 year.

Atmospheric effects occur both at seasonal and at more irregular periods. One of the impressive results obtained from the improved lod data of the last decade is excellent agreement between the short period rotation results after subtraction of the tidal effects, and the atmospheric excitation estimated from meteorological data (see, for example, D.A. Salstein, this volume). The atmospheric effects are estimated from global atmospheric wind and pressure data. These data are integrated over the globe to find the total atmospheric angular momentum as a function of time. The negative time derivatives of these integrated results should be equal to the torque on the mantle, $L_{3}$ in (1), and so can be used to find $\mathrm{m}_{3}$.

Most of the variability in the atmospheric angular momentum is due to changes in zonal winds. The effects of the redistribution of atmospheric mass on the moment of inertia, as inferred from surface pressure observations, are only about $10 \%$ of the effects of winds. There are also likely to be effects due to seasonal variations in the circum-polar 
ocean current around Antarctica. Those effects are probably smaller than the effects of atmospheric pressure, although the estimates are reasonably uncertain.

The most important implication of the good agreement between the lod and atmospheric data is that it suggests existing atmospheric data adequately reflect real global disturbances in the atmosphere. For example, Langley, et al. (1981) found that the inferred atmospheric angular momentum data and the observed lod have a well-correlated 50 day oscillation in common. This has led to atmospheric modelling efforts to understand the source of the oscillation.

\section{POLAR MOTION}

The most pronounced features of the observed polar motion are oscillations at 12 months and 14 months, called the annual wobble and Chandler wobble, respectively. The annual wobble is due to seasonal forcing of the earth by the atmosphere, particularly by the large seasonal pressure variations over central Asia. Since the earth is elliptical rather than spherical, those pressure variations cause a seasonal torque on the earth about an equatorial axis, and that tips the earth causing polar motion. There is also sizable seasonal excitation due to similar torques caused by global changes in ground water storage (Van Hylckama, 1970). The agreement between the observed annual wobble amplitude and the equatorial torques inferred from global pressure and ground water data is good (see Merriam, 1982; Wahr, 1983).

The Chandler wobble is a rotational normal mode of the earth, equivalent to the free nutation of a top. For a rigid earth, the period would be roughly 10 months. The increase of the period to 14 months is due to the ability of the earth to deform : solid earth deformation adds 4 months, oceanic displacements add 1 month, the earth's fluid core subtracts 1 month. Observations of the period have been used to constrain mantle anelasticity at a period of 14 months (see, for example, Smith and Dahlen, 1981). The observed damping time (several decades) has also been used to constrain anelasticity.

The primary excitation source for the Chandler wobble is, however, not well understood. Although there is some disagreement, it appears as though neither earthquakes (through induced changes in the earth's inertia tensor; see eg. Dahlen, 1973) nor the atmosphere and oceans (Wilson and Haubrich, 1976; Wahr, 1983) were the primary excitation source during this century. It may be that the excitation is due to fluid pressure in the core against the core-mantle boundary, as proposed by Le Mouel, et al. (1985). Or, perhaps it is due to irregular fluctuations in global ground water storage. Future VLBI data may help resolve this problem through accurate observations of individual excitation events.

\section{NUTATIONS}

The earth's nutational motion is caused by the gravitational attraction of the sun and moon. The motion can be separated into a discrete sum of periodic terms with frequencies, as seen from the diurnally rotating earth, of one cycle per day modulated by the lunar and solar orbital frequencies.

The earth is believed to have a rotational normal mode, called the free core nutation (FCN), with an eigenfrequency within the diurnal band of nutation frequencies. To 
understand the dynamics of the FCN, suppose the fluid core and solid mantle were tipped about an equatorial axis in opposite directions, and then released. If both the core's internal density distribution and the core-mantle boundary were spherical, there would be no restoring torque, and the core and mantle would remain tipped relative to each other. But, because the real core/mantle boundary is elliptical and the earth is elliptically stratified, there are, instead, restoring pressure and gravitational torques between the core and mantle. As a result, the core and mantle execute periodic twisting motion with respect to each other. This motion is the FCN, and its frequency is one cycle per day plus a factor dependent on the strength of the restoring torque.

In the Appendix, it is shown that under very general conditions (including the possibility that the mantle is not hydrostatically pre-stressed, but not including the effects of a solid inner core) the FCN eigenfrequency is

$$
\omega_{o}=\Omega\left[1+\frac{A}{A_{m}}\left(e_{\mathrm{f}}-\beta\right)\right]
$$

where $A$ and $A_{m}$ are the equatorial principal moments of inertia for the entire earth and the mantle alone; $e_{\mathrm{f}} \cong\left(\mathrm{C}_{\mathrm{f}}-\mathrm{A}_{\mathrm{f}}\right) / \mathrm{A}_{\mathrm{f}}$ is the dynamical ellipticity of the core, where $\mathrm{C}_{\mathrm{f}}$ and $A_{f}$ are the principal moments of the fluid core about the polar and equatorial axes, respectively; and $\beta$ is a numerical factor which represents the effects of deformation and is effectively independent of any aspherical stratification within the earth. The quantity $e_{\mathrm{f}}$ depends on the $\mathrm{Y}_{2}^{0}$ spherical harmonic term of the core internal density field and on the $\mathrm{Y}_{2}^{0}$ component of the shape of the core-mantle boundary. It does not depend on any other spherical harmonic component of the structure. For a hydrostatically prestressed earth, $\beta$ is about $25 \%$ of $e_{\mathrm{f}}$ (Sasao, et al, 1980), and $\omega_{o} \cong(1+1 / 460)$ cycles/day (Wahr, 1981).

The free nutational motion at the period $\omega_{0}$ has never been unambiguously observed. Evidently, it is not excited sufficiently by any interior process. But, because $\omega_{o}$ is so close to the frequencies of the luni-solar nutations, the amplitudes of those nutations are affected by the presence of the mode by up to 20 or 30 milli-arcseconds, a perturbation which can be readily detected even by conventional optical observations of nutation. These effects of the FCN are included in the standard forced nutation model adopted by the IAU, which is based on rigid earth values of Kinoshita (1977) and corrections for non-rigidity from Wahr (1981), the latter assuming a hydrostatically pre-stressed earth.

Because VLBI is tied directly to an inertial reference system, it is superior to any of the other new techniques in resolving the forced nutations. As discussed by T.A. Herring in this volume, the VLBI results disagree with the IAU adopted theory by almost 2 mas, particularly at the annual frequency: $\omega=\Omega(1+1 / 365.25)$ cycles/day. This is the forcing frequency closest to $\omega_{o}$, and it suggests that the FCN frequency may be somewhat larger than expected, close to $\omega_{o}=\Omega(1+1 / 435)$ cycles/day (Gwinn, et al, 1986).

Equation (2) suggests that $\omega_{o}$ can be modified by changing either $\beta$ or $e_{\mathrm{f}}$. Wahr and Bergen (1987) (see, also, Dehant, 1987) considered the effects of mantle anelasticity on $\beta$, and concluded that the corresponding perturbation of $\omega_{0}$ is too small and, more importantly, has the wrong sign. Although the effects of anelasticity are, in principle, 
observable using the current VLBI observations, the larger discrepancy between observation and theory must first be resolved before the VLBI results can be used to learn about diurnal anelasticity.

Probably the most likely explanation for the discrepancy is, instead, uncertainty in $e_{f}$, as postulated by Gwinn, et al. (1986). Recent results based on seismic tomography ( eg. Richards and Hager, 1984; Creager and Jordan, 1986; Morelli and Dziewonski, 1987) suggest that the shape of the core-mantle boundary may diverge appreciably from hydrostatic equilibrium. It is not straightforward to compute the effects on $e_{f}$ given only the shape of the core-mantle boundary. A perturbed boundary and an aspherical density distribution in the mantle will cause perturbations in the internal density surfaces in the core, and these will affect $e_{f}$. But, current models of the boundary based on seismic data suggest values for the non-hydrostatic portion of $e_{f}$ which are as large or larger than the result inferred from the nutation observations. In fact, the VLBI nutation observations are proving to be a valuable independent constraint on the seismic models.

Acknowledgements

The work described in the Appendix was supported by Air Force Geophysical Laboratory contract F19628-86-k-0011, and NASA grant NAG5-485.

\section{REFERENCES}

Alterman, Z., H. Jarosch, and C.L. Pekeris, 1959; Proc. Roy. Soc. London, Ser. A., 252, 80.

Creager, K.C. and T.H. Jordan, 1986; Geophys. Res. Lett., 13, 1497.

Dahlen, F.A., 1973; Geophys. J. Roy. Astr. Soc., 32, 203.

Dehant, V., 1987; Phys. E. Planet. Int., in press.

Gwinn, C.R., T.A. Herring, and I.I. Shapiro, 1986; J. Geophys. Res., 91, 4755.

Kinoshita, H., 1977; Cel. Mech., 15, 277.

Lambeck, K., 1980; The Earth's Variable Rotation: Geophysical Causes and Consequences, Cambridge University Press.

Langley, R.B., R.W. King, I.I. Shapiro, R.D.Rosen, and D.A. Salstein, 1981; Nature, 294, 730.

Le Mouel, J.L., C. Gire, and J. Hinderer, 1985; C.R. Acad. Sc. Paris, 301, 27.

Merriam, J.B., 1982; Geophys. J. Roy. Astr. Soc., 70, 41.

Merriam, J.B., 1985; J. Geophys. Res., 90, 9423.

Munk, W.H., and G.J.F. MacDonald, 1975; The Rotation of the Earth, Cambridge University Press.

Morelli, A. and A.M. Dziewonski, 1987; Nature, 325, 678.

Richards, M.A., and B.H. Hager, 1984; J. Geophys. Res., 89, 5987.

Rochester, M.G., 1984; Phil. Trans. R. Soc. Lond. A, 313, 95.

Sasao, T., S. Okubo, and M. Saito, 1980; in Proceedings of IAU Symposium no. 78 "Nutation and the Earth's Rotation", eds. Fedorov, E., M. Smith, and P. Bender; pub. D. Reidel. 
Smith, M.L., and F.A. Dahlen, 1981; Geophys. J. Roy. Astr. Soc., 64, 223.

Van Hylckama, T.E.A., 1970; in Int. Assoc. Sci. Hydrology, Proc. Reading Symp. World Water Balance, Publ. 92, AIHS-UNESCO.

Wahr, J.M., 1981; Geophys. J. Roy. Astr. Soc., 64, 705.

Wahr, J.M., 1983; Geophys. J. Roy Astr. Soc., 74,451.

Wahr, J., and Z. Bergen, 1986; Geophys. J. Roy. Astr. Soc., 87, 633.

Wilson, C.R., and R.A. Haubrich, 1976; Geophys. J. Roy. Astr. Soc., 46, 707.

Woodhouse, J.H., and F.A. Dahlen, 1978; Geophys. J. Roy. Astr. Soc., 53, 335.

\section{APPENDIX: FCN FREQUENCY FOR A NON-HYDROSTATICALLY PRESTRESSED EARTH}

All existing nutation models assume the earth is hydrostatically pre-stressed. Among the implications of this assumption which could be important for nutation calculations are that, in the unperturbed state, the core-mantle boundary is a surface of constant potential, and that the core-mantle boundary and all internal density surfaces in the core and mantle are elliptical: ie. their departure from spherical symmetry can be described with the single spherical harmonic $\mathrm{Y}_{2}^{0}(\theta, \lambda)$, where $\theta$ and $\lambda$ are co-latitude and eastward longitude, respectively.

Recent results based on seismic tomography suggest that surfaces of constant density in the mantle and surfaces of discontinuity, such as the core-mantle boundary, have shapes which are not consistent with the hydrostatic pre-stress assumption. The $\mathrm{Y}_{2}^{0}$ components of these surfaces differ from the hydrostatic values, and there are large components with other $\mathrm{Y}_{1}^{\mathrm{m}}$ angular dependence. Since the FCN is critically dependent on pressure coupling acting across the core-mantle boundary (and, to a lesser extent, gravitational coupling between the core and mantle), the FCN eigenfrequency is apt to be sensitive to this sort of detail about the surface shapes.

In this Appendix, the FCN eigenfrequency is modelled under only minimal assumptions about the state of pre-stress in the earth. The core is assumed to be hydrostatically pre-stressed (it is, after all, a fluid even at seismic periods), but the mantle is allowed to possess a non-zero deviatoric pre-stress. As a result, the core-mantle boundary and the internal density surfaces in the mantle can have arbitrary shape. Constant density surfaces in the core are uniquely determined by the shape of the core-mantle boundary and by the gravitational potential from the mantle acting on the core. But, the constant density surfaces in the core need not coincide with the surfaces expected for an everywhere hydrostatically pre-stressed earth. The derivation, below, extends the semianalytical model of Sasao, et al. (1980) to account for an initially non-hydrostatic mantle and core-mantle boundary, and to more fully justify a number of the approximations. In this derivation, the inner core is assumed to be fluid.

Consider a reference frame fixed to the mantle and rotating with it. For nutational motion at frequency $\omega_{o}$, the rotation vector of the frame is $\Omega \hat{\mathbf{z}}+\Omega \mathrm{m}_{o}(\hat{\mathbf{x}}+i \hat{\mathbf{y}}) \mathrm{e}^{\mathrm{i} \omega_{o} \mathrm{t}}$, where the nutational motion of the mantle is described by $m_{o}$, and $m_{o} \ll 1$. For the FCN, $\left(\omega_{o}-\Omega\right) / \Omega$ is first order in $\epsilon$, where $\epsilon$ is some measure of the departure of the earth from 
spherical symmetry. The equations of motion in the hydro-statically pre-stressed core for the case of no external forcing are

$$
\begin{gathered}
\rho\left[-\omega_{o}^{2} \mathrm{~s}+2 \mathrm{i} \omega_{o} \Omega \times \mathrm{s}\right]=-\rho \nabla \phi_{1}-\nabla \mathrm{P}_{\mathrm{E}}-\rho_{1} \nabla \Phi-\Omega^{2} \rho \mathrm{m}_{o}\left[(\hat{\mathbf{x}}+\mathrm{i} \hat{\mathrm{y}}) \mathrm{z}\left(\Omega-\omega_{o}\right)+\right. \\
\left.+\hat{\mathbf{z}}(\mathrm{x}+\mathrm{iy})\left(\Omega+\omega_{o}\right)\right] \\
\nabla^{2} \phi_{1}=4 \pi \mathrm{G} \rho_{1}
\end{gathered}
$$

where 8 is the displacement field in the core; $\phi_{1}, \mathrm{P}_{\mathrm{E}}$, and $\rho_{1}$ are the Eulerian perturbations in gravitational potential energy, pressure, and density, respectively; $\Omega=\Omega \hat{\mathbf{z}}$ represents the mean rotation of the earth; and $\rho$ and $\Phi$ are the initial density and total potential energy (gravitational plus centrifugal) and can have arbitrary angular dependence, except that surfaces of constant $\rho$ must be surfaces of constant $\Phi$ in the core. There are, also, equations relating $\rho_{1}$ and $\mathrm{P}_{\mathrm{E}}$ to derivatives of 8 , which are not shown here. And, there are similar, although more complicated, differential equations describing displacements in the mantle, which are also omitted here. The mantle equations explicitly include the non-hydrostatic pre-stress (see Woodhouse and Dahlen, 1978, for both the mantle and core equations).

For nutational motion, the displacement field in the core has the form

$$
\mathbf{s}(\mathbf{r})=\Omega \theta_{o}(\hat{\mathbf{x}}+\mathrm{i} \hat{\mathbf{y}}) \times \mathbf{r}+\mathbf{s}_{\mathrm{d}}(\mathbf{r})
$$

where $\theta_{0}$ represents the mean nutational motion relative to the mantle, and $s_{\mathrm{d}}$, which represents the deformation of the core, is first order in $\epsilon$ compared with the $\theta_{o}$ term. Taking ( $\hat{\mathbf{x}}-\mathrm{i} \hat{\mathbf{y}}) \cdot[\mathbf{r} \times($ eqn.(A1)) $]$ and integrating through the core, and using (A2) for $\mathbf{8}$, gives an angular momentum balance equation for the core:

$$
\begin{aligned}
& 2 i \Omega^{2} A_{f} m_{o}+2 \omega \Omega^{2} A_{t} \theta_{o} \frac{\Omega-\omega_{o}}{\Omega}+i \omega_{o} \Omega c_{-}^{\mathrm{f}} \\
& =(\hat{x}-i \hat{y}) \cdot \int_{\text {core }} \mathbf{r} \times\left[-\rho \nabla \phi_{1}-\nabla P_{E}-\rho_{1} \nabla \Phi\right]
\end{aligned}
$$

where $A_{f}$ is the core's principal moment of inertia in the equatorial plane; and $c_{-}^{f}=c_{13}^{f}-i c_{23}^{f}$, where the $c_{i j}^{f}$ represent the perturbation in the core's inertia tensor due to the motion. The terms on the right hand side of (A3) include the pressure and gravitational torques on the core from the mantle.

All terms in (A3) are second order in $\epsilon$ or smaller, given that $\theta_{0}$ is zero order. For example, $\phi_{1}$ is first order since it is a deformation term, and the integral of $\rho \mathrm{r} \times \nabla \phi_{1}$ is 0 no matter what $\phi_{1}$ is, unless either the core shape or the internal density in the core is aspherically symmetric. As another example, $\Omega \omega_{0} \cong \Omega^{2}$ is a first order quantity (the unperturbed centrifugal force is order $\epsilon$ times the unperturbed gravitational force) as is $\left(\Omega-\omega_{0}\right) / \Omega$. Thus, the $\theta_{0}$ term in (A3) is second order. As a final example, $m_{o}$ is first order compared with $\Omega \theta_{o}\left(\mathrm{~m}_{o}\right.$ represents motion of the mantle rotation axis as seen from the mantle and is of order $\left.\left(\Omega-\omega_{o}\right) \theta_{o}\right)$ so that $\Omega^{2} \mathrm{~m}_{o}$ is second order.

A comparable angular momentum equation for the entire earth, after dropping all terms third order or smaller, is

$$
2 \mathrm{iAm}_{0}=2\left(\omega_{0}-\Omega\right) \mathrm{A}_{\mathrm{f}} \theta_{0}
$$


where $\mathbf{A}$ is the principal moment of inertia for the entire earth. (This result is consistent with the assumption that $m_{o}$ is first order.)

Next, $c_{-}^{f}$ and the right hand side of (A3) can be related to $\theta_{o}$. This is done by using (A2) in (A1), dropping all terms second order or smaller in $\epsilon$ which occur in (A1) and in the corresponding mantle differential equations and in all boundary conditions, and then solving the entire system on a computer. In the core, for example, the first order (spheroidal) equations for $\phi_{1}, \mathrm{P}_{\mathrm{E}}$, and $\rho_{1}$ reduce to

$$
\rho_{0} \nabla \phi_{1}+\nabla \mathrm{P}_{\mathrm{E}}+\rho_{1} \nabla \Phi_{0}=\left(\mathrm{i} \theta_{0} \Omega^{3} \sqrt{8 \pi / 15}\right) \rho_{0} \nabla\left(\mathrm{r}_{2} \mathrm{Y}_{2}^{1}\right)
$$

where $\rho_{0}$ and $\Phi_{0}$ are the spherically symmetric parts of $\rho$ and $\Phi$. No $m_{o}$ terms are included in (A5), because those terms are second order.

The corresponding first order differential equations in the mantle are the usual set of equations describing deformation of a spherical, non-rotating, static, and hydrostatically pre-stressed mantle (see, for example, Alterman, et al., 1959), with no first order apparent force corresponding to the $\theta_{0}$ term in (A5). Although there is an apparent force in the mantle which depends on $\mathrm{m}_{o}$, that force is second order. All first order boundary conditions within the core and the mantle and at the outer surface are also equivalent to the boundary conditions for a spherical, non-rotating, hydrostatically prestressed earth.

In effect, then, all deformation-related quantities can be computed by solving the static equations of motion for a spherical, non-rotating, hydrostatically pre-stressed earth, subject to an apparent force proportional to $\rho_{0} \nabla\left(\mathrm{r}^{2} \mathrm{Y}_{2}^{1}\right)$ in the core and equal to 0 in the mantle. Note that, as a consequence, neither the non-hydrostatic pre-stress in the mantle nor any of the earth's aspherical structure enters explicitly into any of the first order deformation equations. Their effects are included only through the integrals on the right hand side of (A3).

Because the apparent force in the core is proportional to $\rho_{0} \nabla\left(r^{2} Y_{2}^{1}\right)$, and because the first order deformation equations are spherically symmetric, $\rho_{1}, \mathrm{P}_{\mathrm{E}}$, and $\phi_{1}$ have $\mathrm{Y}_{2}^{1}(\theta, \lambda)$ angular dependence. Using this angular dependence, (A5) yields directly

$$
\begin{gathered}
\mathrm{P}_{\mathrm{E}}(\mathrm{r}, \theta, \lambda)=\rho_{0}(\mathrm{r})\left[\left(\mathrm{i} \Omega^{3} \theta_{0} \sqrt{8 \pi / 15}\right) \mathrm{r}^{2} \mathrm{Y}_{2}^{1}(\theta, \lambda)-\phi_{1}(\mathrm{r}, \theta, \lambda)\right] \\
\rho_{1}(\mathrm{r}, \theta, \lambda)=\frac{\partial_{\mathrm{r}} \rho_{0}(\mathrm{r})}{\partial_{\mathrm{r}} \Phi_{0}(\mathrm{r})}\left[\phi_{1}(\mathrm{r}, \theta, \lambda)-\left(\mathrm{i} \Omega^{3} \theta_{0} \sqrt{8 \pi / 15}\right) \mathrm{r}^{2} \mathrm{Y}_{2}^{1}(\theta, \lambda)\right]
\end{gathered}
$$

Using (A6) in (A3) and doing the integrals gives a result accurate to second order of

$$
2 \mathrm{i} \Omega^{2} \mathrm{~m}_{o}+2 \Omega^{2} \theta_{o}\left(\Omega-\omega_{o}\right)+\frac{i c_{-}^{\mathrm{f}} \Omega^{2}}{\mathrm{~A}_{\mathrm{f}}}=-2 \Omega^{3} \theta_{o} \mathrm{e}_{\mathrm{f}}
$$

where $e_{f}$ is the dynamical ellipticity of the core and is equal to $\left(C_{f}-A_{f}\right) / A_{f}$, where $C_{f}$ is the polar moment of inertia of the core.

Define the dimensionless, real parameter $\beta$ so that

$$
\mathrm{c}_{-}^{\mathrm{f}}=\beta 2 \mathrm{~A}_{\mathrm{f}} \mathrm{i} \Omega \theta_{0}
$$

Here, $\beta$ can be determined by solving the deformation equations on a computer. Using 
(A4) and (A8) in (A7) and solving for the FCN eigenfrequency, $\omega_{o}$, gives

$$
\omega_{o}=\Omega\left[1+\frac{A}{A_{m}}\left(e_{f}-\beta\right)\right]
$$

where $A_{m}$ is the principal moment of inertia of the mantle.

Although the result (A9) was derived here without assuming a hydrostatically prestressed mantle, it is identical in form to the hydrostatic result. The dynamical ellipticity, $e_{f}$, depends on the $Y_{2}^{0}$ component of the core-mantle boundary shape, and on the $\mathrm{Y}_{2}^{0}$ terms in the density stratification inside the core. There is no dependence, to this order of approximation, on any other $\mathrm{Y}_{1}^{\mathrm{m}}$ terms in the aspherical structure. The factor $\beta$ represents the effects of deformation and is insensitive, to this order, to aspherical structure. For a hydrostatically pre-stressed earth, $\beta$ is about $25 \%$ of $e_{f}$, and $\omega_{0} \cong\left(1+\frac{1}{460}\right)$ cycles per day. 